\section{ORIGINAL RESEARCH}

A. Scollato

R. Tenenbaum

G. Bahl

M. Celerini

B. Salani

N. Di Lorenzo

\title{
Changes in Aqueductal CSF Stroke Volume and Progression of Symptoms in Patients With Unshunted Idiopathic Normal Pressure Hydrocephalus
}

BACKGROUND AND PURPOSE: Idiopathic normal pressure hydrocephalus (iNPH) represents a diagnostic challenge, given its variable presentation and progression. Stroke volume (SV), defined as the mean volume of CSF passing through the aqueduct during both systole and diastole, greater than or equal to $42 \mu \mathrm{L}$, serves as a selection criterion for patients with good probabilities of improvement after ventriculoperitoneal shunt surgery (VPS). In this study, we evaluated the changes in SV during the progression of clinical symptoms in patients with suspected NPH.

MATERIALS AND METHODS: Nine patients who presented with clinical and radiologic evidence of NPH, but refused treatment with VPS, were evaluated every 6 months for up to 2 years for progression in their clinical symptoms and changes in their SV, as measured by phase-contrast cine MR imaging (PCCMR).

RESULTS: SV seems to increase between the onset of the symptoms and the following 18 to 20 months, then seems to plateau, followed in the next 18 to 20 months by a slight decline, and finally to a more precipitous drop in the next 12 months. During this time, however, the patient's clinical symptoms progressively worsen.

CONCLUSION: Patients with a low SV have not necessarily had brain atrophy and can show, in the following months, a progressive increase in SV, which qualifies them as good candidates for VPS. The progressive reduction of the SV in untreated patients with worsening clinical symptoms may be a sign of a progressive cerebral ischemic injury, which renders the NPH irreversible.

$\mathbf{T}$ he syndrome of progressive mental deterioration and neurologic disturbances including psychomotor retardation, gait unsteadiness, and incontinence of urine associated with hydrocephalus, in the setting of normal CSF pressure on lumbar puncture and the absence of papilledema, was coined normal pressure hydrocephalus (NPH) in $1965 .{ }^{1}$ To this day, the exact cause of the idiopathic form of NPH (iNPH) remains unclear. However, it is generally agreed on that on the basis of $\mathrm{NPH}$, there is an abnormal absorption of CSF with a consequent accumulation of CSF in the ventricular space. For at least the last 20 years, the mainstay of therapy for NPH has been diversion of CSF via a ventriculoperitoneal shunt (VPS). ${ }^{2,3}$ iNPH typically manifests in elderly people, and its correct diagnosis is complicated by the variability of its clinical presentation and progression. iNPH can resemble, or occur in combination with, various disorders such as cerebrovascular disease, neurodegenerative disorders (eg, Alzheimer disease, Parkinson disease, Lewy body disease), primary urologic disorders, and spinal stenosis. Not all patients initially diagnosed with NPH improve after VPS, which may be because of misdiagnosis $^{4}$ or because the disease has progressed to a stage of irreversible neurodegeneration. In clinical practice, a careful

Received May 16, 2007; accepted after revisions July 3.

From the Departments of Neurosurgery (A.S., R.T., N.D.) and Radiology (M.C.), and Emergency Department (B.S.), Geriatric Agency, University of Florence, Florence, Italy; and Department of Radiology (G.B.), University of California, San Diego, San Diego, California.

Please address correspondence to Antonio Scollato, PhD, Department of Neurosurgery, University of Florence, Largo P. Palagi, 1, 50139 Florence, Italy; email: a.scollato@ neurochirurgiafirenze.it

DOI 10.3174/ajnr.A0785 selection of patients for surgery is the main challenge of this syndrome.

MR imaging has been proposed to quantify the flow of CSF in the aqueduct. ${ }^{5,6}$ By phase-contrast cine MR imaging (PC$\mathrm{CMR}$ ), the stroke volume (SV), defined as the mean volume passing through the aqueduct during both systole and diastole, can be calculated. A SV greater than or equal to $42 \mu \mathrm{L}$ serves as a selection criterion for patients with good probabilities of improvement after VPS. ${ }^{7}$ The goal of this work is to analyze the changes in $\mathrm{SV}$ and the progression of symptoms in patients diagnosed with iNPH who refused treatment with VPS.

\section{Materials and Methods}

\section{Selection of Patients}

Between March 2000 and April 2004, we examined 9 patients (5 women, 4 men; mean age, 74.8 years) presenting with symptoms and MR imaging findings suggestive of NPH. All patients had widening of the ventricles (Evans ratio $>0.30$ ) (Fig 1 ) and at least 2 of the classic triad of neurologic disturbances associated with NPH. Seven patients presented at the first evaluation with an increased aqueductal SV. In the remaining 2 patients, increased SV was noted at the first follow-up session after 6 months. In accordance with our NPH protocol of the study, we proposed treatment with a VPS. After the risks and benefits of treatment were explained to the patients or their surrogate decision makers, the 9 patients in our study refused a VPS. We repeated the CSF aqueductal dynamic study and clinical evaluation of these patients every 6 months for a total of 2 years. 


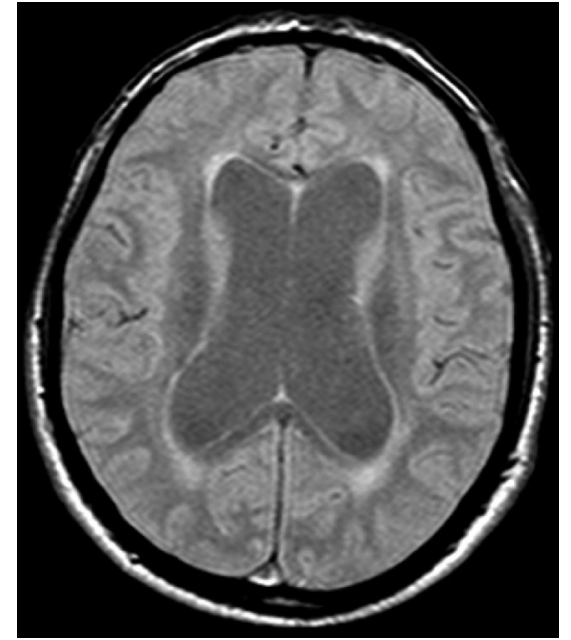

Fig 1. Ventricular enlargement in MR image of a patient with NPH with a positive Evans Index.

\begin{tabular}{lccc}
\hline $\begin{array}{l}\text { Table 1: Symptoms present at first evaluation and duration of } \\
\text { presence of each of these symptoms since their onset }\end{array}$ \\
\hline \multicolumn{4}{c}{$\begin{array}{c}\text { Duration (mos.) of Symptoms at First } \\
\text { Evaluation }\end{array}$} \\
\cline { 2 - 4 } Patient No. & Gait & Urinary & Cognitive \\
\hline 1 & 11 & 0 & 7 \\
2 & 6 & 4 & 6 \\
3 & 18 & 8 & 6 \\
4 & 30 & 10 & 18 \\
5 & 10 & 0 & 6 \\
6 & 12 & 7 & 0 \\
7 & 6 & 2 & 12 \\
8 & 12 & 6 & 12 \\
9 & 5 & 1 & 0 \\
\hline
\end{tabular}

\section{Clinical Evaluation}

All 9 patients were clinically diagnosed with NPH on the basis of the cardinal symptoms of gait disturbance, cognitive impairment, and urinary bladder urgency or incontinence. We evaluated mental disturbances using the Mini-Mental State Examination (MMSE). ${ }^{8}$ Urinary disorders were evaluated by the Urinary Incontinence Scale (UIS) graded into 4 categories: normal urinary function (grade 0 ), urgent urination or sporadic incontinence (grade 1), frequent incontinence (grade 2), and complete incontinence (grade 3). Gait disorders were evaluated by the Gait Scale (GS) graded into 4 categories: normal gait (grade 0); discrete imbalance when turning with short steps, widened base, and occasional falling (grade 1); frequent falls and aid needed for ambulation (grade 2); and impossible gait (grade 3 ). We used the same criteria for follow-up evaluations. The symptoms that were present at the first evaluation and the duration of the presence of each of these symptoms since their onset were recorded for each patient (Table 1).

\section{PCCMR Technique for Dynamic Flow Measurements at the Level of the Aqueduct}

We conducted the study with a $0.5 \mathrm{~T}$ superconductive MR scanner (Philips, Best, the Netherlands) using a PCCMR pulse sequence (TR, $54 \mathrm{~ms}$; TE, 9-13 ms; NEX, 2; matrix, $190 \times 256$ pixels; section thickness, $5 \mathrm{~mm}$; FOV, $12 \mathrm{~cm}$ ). A cine acquisition with sensitivity to velocity in the section select direction was obtained on an oblique-axial plane of section perpendicular to the aqueduct (at the inferior collicu- lus) just above the fourth ventricle (Fig 2). In addition to its course being nearly vertical at this location, the area of the aqueduct is larger in this region, minimizing partial volume averaging. The strength of the velocity encoding is parameterized by the velocity that produces a phase shift of $180^{\circ}$ between the acquisitions (Venc). Stronger encoding (smaller Venc values) produces improved precision for measuring slow motion but accommodates a smaller range of velocities before aliasing occurs. In our study, Venc was always selected so as to avoid velocity aliasing in regions of interest. This PCCMR method produces conventional magnitude and phase-contrast flow images, the former from the average of magnitude reconstruction of the 2 datasets at each point in time and the latter from the difference in phase images.

We interpolated the acquired raw data to produce 16 frames equally spaced in the cardiac cycle by means of plethysmography with retrospective cardiac gating. The Venc value ranged between 15 and $20 \mathrm{~cm} / \mathrm{s}$. A phase-contrast cine produces images that represent velocity as a function of time in the cardiac cycle. Before we performed quantitative analysis of flow within a region of interest, we estimated a constant baseline for that region by calculating the average throughout the cardiac cycle of the apparent velocity in a neighboring static structure. These baseline regions were chosen where brain motion is minimal or absent during the cardiac cycle, such as the middle cerebellar peduncle. In addition, because the baseline measurement was averaged throughout the cardiac cycle and because there is no net brain motion, cyclical brain motion does not corrupt the baseline estimate or alter the measured pulsatile waveform.

For each pixel in each of the 16 frames, the product of the velocity in the pixel (apparent velocity minus the baseline) and the pixel area is an estimate of the volumetric flow rate (VFR) (milliliters per second) through the pixel. VFR, as a function of time through the CSF aqueduct, was measured as the sum of the VFRs through the pixels within a defined region of interest for each frame. The imaging plane was positioned perpendicular to the flow rate through the cycle and was expressed as milliliters per minute or as milliliters per cycle, by dividing by the heart rate. The accuracy of average VFR measurements has been validated both in vitro ${ }^{9}$ and in vivo. ${ }^{10}$ For a given region of interest in any frame, the product of VFR (milliliters per second) and the time interval between cine frames (milliseconds) is the volume (microliters) displaced through the region of interest during that frame. The sum of this quantity over all frames in which it is positive is an estimate of the volume that is displaced in 1 direction (caudocranial direction in diastole) through the region of interest. A similar sum over negative values can be performed (craniocaudal direction in systole).

In our study, SV was the average of systolic and diastolic volumes passing through the aqueduct. The area of measurement ranged from $0.068 \mathrm{~cm}^{2}$ to $0.136 \mathrm{~cm}^{2}$ in size and comprised at least 13 pixels. Errors in measurement as a result of partial volume averaging were minimized by orienting the section perpendicular to the aqueduct and because the pixel size was small relative to the aqueduct. A Venc of 15 to $20 \mathrm{~cm} / \mathrm{s}$ allowed measurement without aliasing.

\section{Results}

After the first evaluation, clinical progression and SV measurements were re-evaluated every 6 months for a total of 24 months in 5 patients, 18 months in 2 patients (patient 2 did not cooperate with follow-up given his progressive deterioration of symptoms, and patient 4 died 20 months after the first evaluation from cardiovascular complications), and 12 months in the remaining 2 patients (patients 7 and 8 did not cooperate with follow-up because of progressive symptomatic 

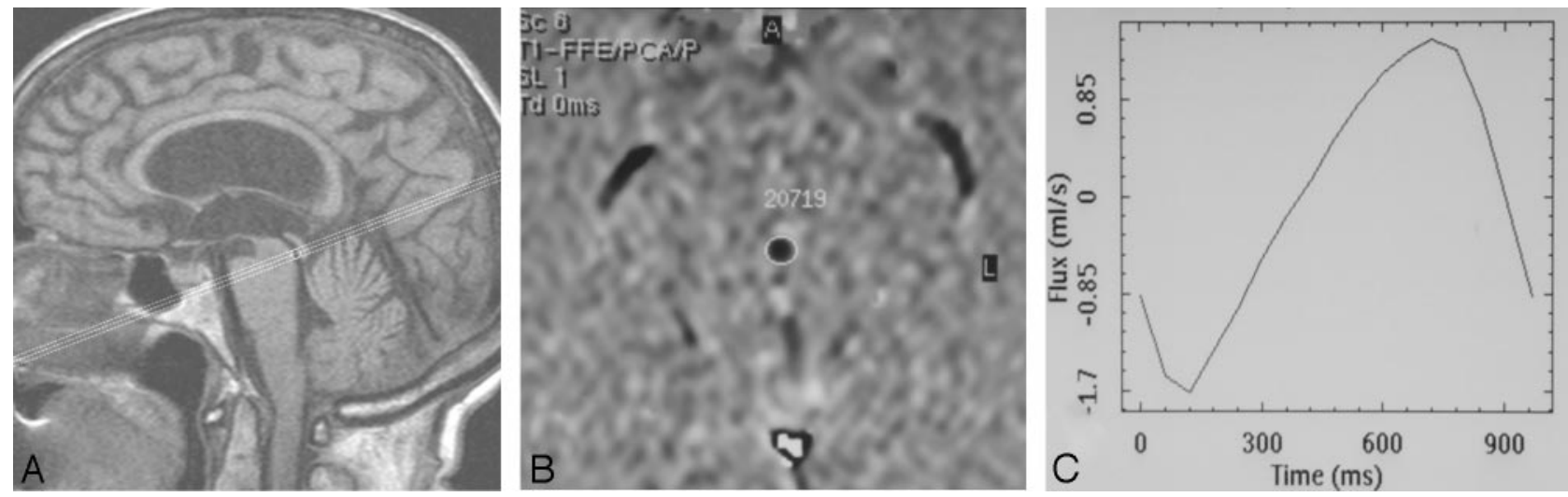

Fig 2. PCCMR imaging CSF dynamic study in a 74-year-old patient with NPH. A, Midline sagittal T1-weighted MR imaging is used to graphically describe the phase-contrast cine series. The section is placed at the level of the inferior colliculus, perpendicular to a line drawn through the distal aqueduct. $B$, Axial section in which region of interest is drawn as close as possible to the aqueduct border. C, Respective absolute values of CSF during 16 cardiac phases are reported on the graph. The flow plot demonstrates sinusoidal pattern of flow where negative values represent aqueductal systolic CSF volume (microliter) outflow and positive values represent diastolic CSF volume inflow.

\begin{tabular}{|c|c|c|c|c|c|c|c|c|c|c|c|c|c|c|c|}
\hline \multirow[t]{2}{*}{ Patient No. } & \multicolumn{5}{|c|}{ GS (mos.) } & \multicolumn{5}{|c|}{ UIS (mos.) } & \multicolumn{5}{|c|}{ MMSE (mos.) } \\
\hline & 0 & 6 & 12 & 18 & 24 & 0 & 6 & 12 & 18 & 24 & 0 & 6 & 12 & 18 & 24 \\
\hline 1 & 1 & 1 & 2 & 2 & 2 & 0 & 2 & 2 & 1 & 2 & 27 & 25 & 19 & 20 & 17 \\
\hline 2 & 1 & 1 & 1 & 2 & NE & 1 & 1 & 1 & 2 & $\mathrm{NE}$ & 16 & 16 & 15 & 16 & $\mathrm{NE}$ \\
\hline 3 & 2 & 2 & 2 & 2 & 2 & 2 & 2 & 2 & 2 & 3 & 18 & 17 & 16 & 13 & 13 \\
\hline 4 & 2 & 2 & 2 & 2 & NE & 2 & 2 & 3 & 3 & $\mathrm{NE}$ & 17 & 18 & 15 & 13 & $\mathrm{NE}$ \\
\hline 5 & 1 & 1 & 1 & 2 & 2 & 0 & 0 & 1 & 2 & 1 & 26 & 24 & 23 & 23 & 22 \\
\hline 6 & 1 & 1 & 2 & 2 & 3 & 2 & 3 & 2 & 2 & 3 & 29 & 27 & 25 & 22 & 18 \\
\hline 7 & 1 & 1 & 2 & NE & NE & 1 & 1 & 2 & $\mathrm{NE}$ & $\mathrm{NE}$ & 21 & 21 & 18 & NE & $\mathrm{NE}$ \\
\hline 8 & 1 & 1 & 1 & NE & NE & 1 & 2 & 2 & $\mathrm{NE}$ & $\mathrm{NE}$ & 19 & 19 & 17 & $\mathrm{NE}$ & $\mathrm{NE}$ \\
\hline 9 & 1 & 1 & 1 & 1 & 2 & 0 & 0 & 1 & 1 & 2 & 27 & 27 & 25 & 22 & 18 \\
\hline
\end{tabular}

Note:-GS indicates Gait Scale; UIS, Urinary Incontinence Scale; MMSE, Mini-Mental State Examination; NE, not evaluated.

Table 3: SV at first evaluation and after 6, 12, 18, and 24 months for each patient

\begin{tabular}{lrrrrr}
\hline Patient No. & \multicolumn{5}{c}{ CSF Aqueductal SV (mos.) } \\
\hline & 0 & 6 & 12 & 18 & 24 \\
1 & 74 & 162 & 187 & 127 & 112 \\
2 & 127 & 147 & 133 & 65 & $\mathrm{NE}$ \\
3 & 67 & 76 & 75 & 98 & 49 \\
4 & 54 & 25 & 20 & 21 & $\mathrm{NE}$ \\
5 & 77 & 107 & 94 & 68 & 30 \\
6 & 23 & 186 & 165 & 86 & 75 \\
7 & 57 & 95 & 104 & $\mathrm{NE}$ & $\mathrm{NE}$ \\
8 & 48 & 73 & 85 & $\mathrm{NE}$ & $\mathrm{NE}$ \\
9 & 28 & 65 & 154 & 145 & 86 \\
\hline
\end{tabular}

Note:-SV indicates stroke volume; NE, not evaluated.

deterioration). The clinical data at the first evaluation and after $6,12,18$, and 24 months are reported in Table 2.

The respective SV data at the first evaluation and after 6, 12, 18 , and 24 months are reported in Table 3 and are represented graphically in Fig 3. Considering the time of onset of the reported first symptoms of NPH in each patient, we have standardized the SV curves reported in Table 1 (Fig 4).

\section{Discussion}

NPH represents one of the few treatable causes of dementia, in which neurologists, neurosurgeons, and neuroradiologists work together to make the diagnosis. NPH is difficult to diagnose, given its variable presentation and progression as well as its overlap and superimposition with other neurologic disorders. After a diagnosis is made, it is still difficult to identify the patients who are likely to show symptomatic improvement with VPS, the so-called shunt-responders. It is important to accurately identify potential shunt-responders because complications associated with VPS occur $13 \%$ to $50 \%$ of the time and may further worsen the patient's quality of life. ${ }^{11}$ To clarify the cause of NPH, make an accurate diagnosis of NPH, and appropriately identify the shunt-responders, several tests have been proposed, including cisternogram, ${ }^{12}$ tap test, ${ }^{13,14}$ resistance measures ${ }^{15}$ external lumbar drainage, ${ }^{14,16,17}$ and intracranial pressure recording. ${ }^{17}$

Much research has focused on the development of a useful, noninvasive prognostic test for NPH. Since its introduction, MR has been shown to have numerous advantages in the evaluation of patients with suspected NPH. ${ }^{18,19}$ On proton-attenuation-weighted images, cerebral aqueductal CSF flow void, the loss of signal intensity as a result of increased CSF velocity in the cerebral aqueduct, was described in patients with clinical NPH. ${ }^{18}$ In 1991, Bradley ${ }^{20}$ found a significant correlation between the major extension of flow void and response to VPS. Mascalchi et $\mathrm{al}^{21}$ obtained the same results in 1993. However, 5 years later Bradley ${ }^{7}$ noted that the appearance of the flow void is highly dependent on the acquisition parameters used, as well as on the technical characteristics of the MR imaging system (eg, gradient strength). In addition, evaluation of the flow void is subjective, and common use of gradient moment nulling or "flow compensation" makes the flow void more difficult to evaluate, possibly rendering the flow void a less sensitive predictor than originally reported for nonflowcompensated images. ${ }^{7,20}$ To rectify these shortcomings, Brad- 

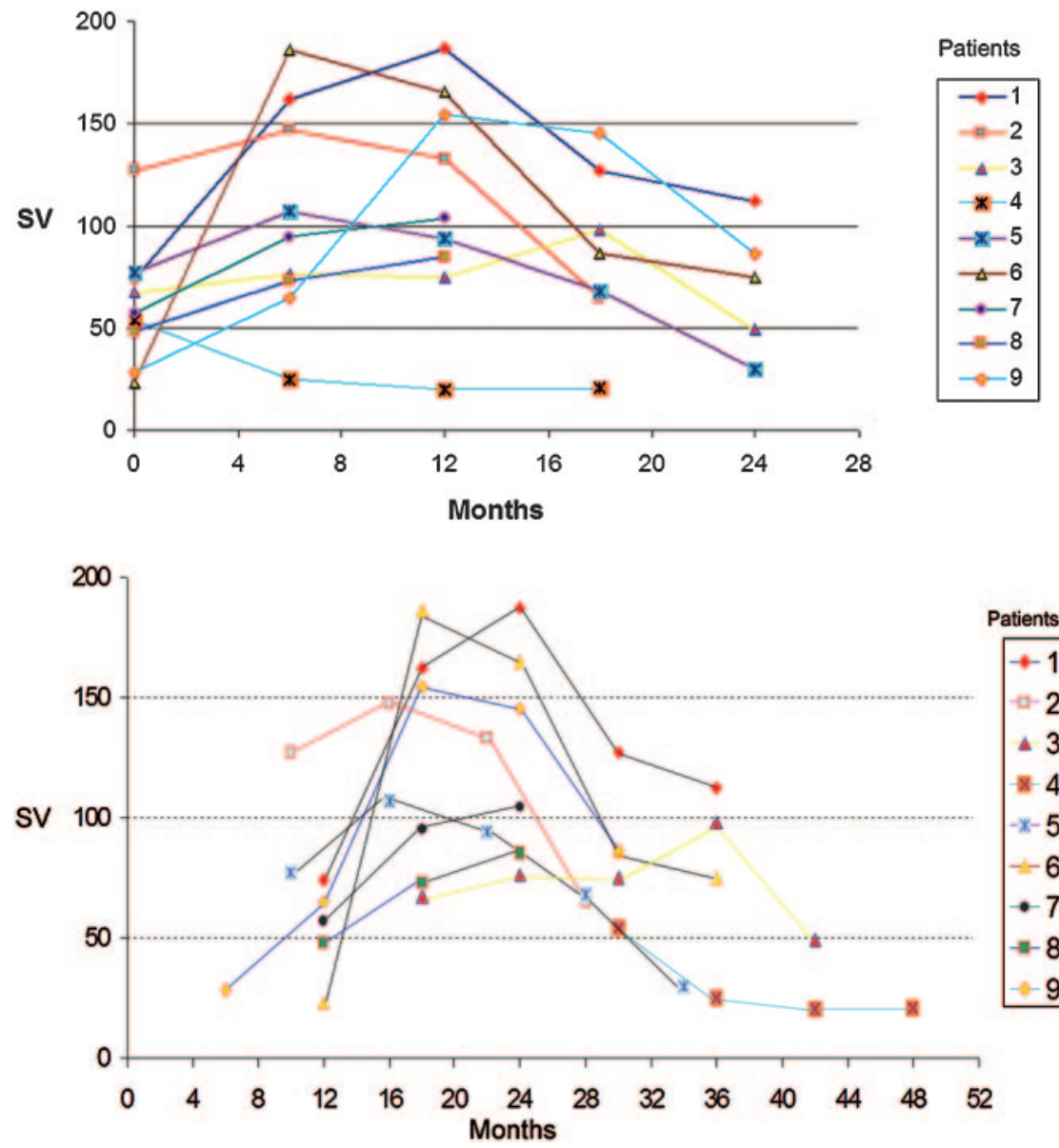

Fig 3. Changes in SV values at $6,12,18$, and 24 months for each of the 9 patients.
Fig 4. Changes in SV values (Table 1) standardized for the estimated onset of NPH, as per the reported first symptoms of NPH. ley replaced the qualitative with a quantitative evaluation of the aqueductal CSF dynamic. Considering the pulsatility and velocity of CSF, previously shown by phase-contrast velocity MR imaging performed with retrospective cardiac gating, ${ }^{23}$ Bradley $^{7}$ imposed a criterion for selection of patients with good probabilities for improvement after VPS as having a SV (mean volume of CSF passing through the aqueduct during both systole and diastole) of $42 \mu \mathrm{L}$ or more.

In our study, we used a similar, yet not identical, technique to that described by Bradley. Because the technique is not exactly the same, the SV cutoff for shunt-responsive NPH may be slightly different, though SV is likely to vary slightly based on equipment and technique.

Nine patients with clinical and MR signs diagnostic of iNPH demonstrated a constant worsening of symptoms. Although the urinary and gait scales could not be considered continuous variables, we used the mean value to describe the progression of these disturbances. At the first observation, gait impairment was present in all 9 patients (100\% of cases). Seven patients presented with a discrete imbalance when turning with short steps, and 2 patients (patients 3 and 4) presented with frequent falls and required aid for ambulation. In these 2 patients, clinical presentation of the disease lasted at least 18 months before the first evaluation. After 12 months, gait worsened in 3 patients. After 24 months, gait worsened in 4 of the 5 patients who were followed up to this time. One patient (patient 3) remained at GS 2 from initial presentation to follow-up at 24 months. The gait disorder was present in all the patients $(100 \%)$ at the first evaluation, and it worsened further in the next months. The mean GS at the first evaluation was 1.2 , which progressed to a GS of 2.2 at 24 months $(P=$ $.03)$.

Cognitive impairment was reported in 5 (56\%) of 9 patients at the first evaluation. The mean MMSE at the first evaluation was 22 of 30 . After 12 months, cognitive impairment worsened in 8 of 9 patients, with a mean decrease of 2.6 points $(P=.02)$. After 24 months, cognitive impairment worsened in all 5 patients who were followed up to this time, with a mean MMSE decrease of 7.8 points $(P=.005)$, from 25.4 at the first evaluation to 17.6 at 24 months.

Urinary disorders were present in $6(67 \%)$ of 9 patients at the first evaluation. By 12 months, all patients reported urinary dysfunction. All patients had increased UIS at their final evaluation compared with their respective first evaluation. At the first evaluation, the mean UIS was 1.0; after 24 months, it was $2.2(P=.005)$.

In general, with a progressive worsening of the symptoms of NPH, SV seems to increase between the onset of the symptoms and the following 18 to 20 months, then plateaus.

According to Bradley's hypothesis, ${ }^{20}$ it is suspected that there is space surrounding the normal brain during diastole, which is occupied by CSF in the subarachnoid spaces and cortical veins. In addition, there is space within the brain itself, which is occupied by CSF in the ventricles. During systole, blood flows into the brain under arterial pressure, causing inward and outward expansion. The inward expansion compresses the ventricles, leading to outflow of CSF, which we observe as a normal aqueductal SV (Fig $5 A$ ). In early commu- 

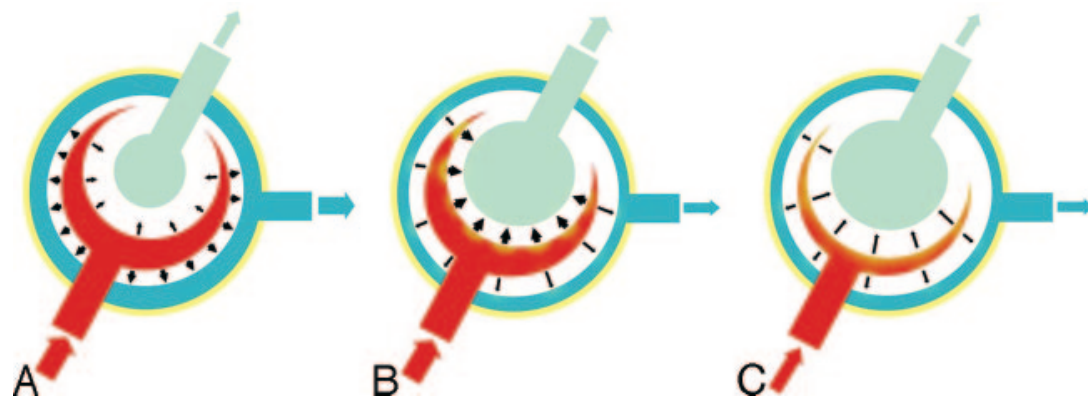

Fig 5. Proposed model demonstrating SV changes in NPH. A In healthy subjects, expansion of the cerebral hemispheres occurs both outward and inward. The outward expansion produces venous blood outflow as a result of compression on the cortical veins. Inward expansion produces flow of CSF into the aqueduct as a result of compression of the lateral and third ventricles. $B$, In communicating hydrocephalus, the brain has already expanded outward during diastole, compressing the cortical veins. However, during systole, with arterial blood entering, the systolic expansion is directed inwards, resulting in a much greater $S V$ in the aqueduct. $C$, Progressive ischemia and a reduction of arterial inflow results in a decreased "ventricular CSF pump." nicating hydrocephalus, the same volume of brain tissue is compressed against the inner table of the calvaria during diastole. When blood flows into the brain during systole, all systolic expansion is directed inward, compressing the enlarged ventricles, leading to a much greater outflow of CSF, which causes an increased aqueductal SV (Fig 5B). The increased volume of the ventricles produces a continuous compression and a tangential shearing force on the brain parenchyma. In time, these forces create a pressure-shearing gradient in the brain around the ventricles. The paracentral fibers are the first to be affected, which is why the first symptom that patients usually experience is gait disturbance. As a result of the persistent compression and shearing of the subcortical and cortical convexities, with time, these patients experience dementia and urinary incontinence.

After the plateau period (18-20 months), SV declines slightly then drops more precipitously in the next 12 months. During systole, the cerebral arterial inflow constitutes the "CSF pump" and creates the "ventricular squeezing," which leads to flow of CSF into the aqueduct. With progression of the disease, the forces produced by the progressive ventricular enlargement lead to vascular stretching and compression. ${ }^{24}$ As a result, progressive ischemia occurs in the brain parenchyma, initially in the periventricular white matter (PWM), which then extends to the cortex and the thalamus. ${ }^{25}$ During this initial stage of ischemia, a marked dilation of the cerebral arterioles occurs, and as demonstrated by Miyake et $\mathrm{al},{ }^{26}$ the administration of acetazolamide in this stage does not induce further dilation given the extensive dilation that has already occurred. The extensive arterial dilation promotes movement of water from the vascular compartment to the interstitial space. This water flow adds to the transependymal flow of CSF, mainly into the paraventricular region, increasing the hydrocephalic edema. This further increases the cerebral parenchymal rigidity, which opposes the "ventricular squeezing" during each and every cardiac cycle. In addition, the same edema within the cerebral parenchyma constitutes an additional volume that reduces the cerebral compliance as the periventricular-cortical disease progresses. In addition, the arteriolar dilation decreases vascular compliance, which leads to a decreased pulsatile force and blood flow (Fig 5C), as well as an increased susceptibility of the dilated arterioles to the external compression. These mechanisms result in arteriolar collapsing and a significant worsening of the cerebral ischemia.

We believe that the continuous decrease of the SV may be an expression of a major cerebrovascular insult that, in time, will induce chronic cerebral ischemic disease. At this stage, patients develop cerebral atrophy, which decreases brain mass.
With increasing atrophy, these patients are likely to become less responsive to VPS.

The above-outlined mechanism has been described in the past by Bradley ${ }^{22}$ in a letter to Kitikaghi. In his letter, Bradley refers to the dynamic changes in the CSF as an aqueductal flow void, whereas in our study we regard them as related to SV. In the same letter, Bradley reported that if an increased flow void is not seen in an appropriately symptomatic patient, there could be several explanations, such as the patient had already developed central atrophy and will no longer respond to the shunt, or the MR technique is not sensitive enough to detect hyperdynamic flow. In addition to these 2 observations, we propose a third, in which patients with a normal SV initially may worsen and express increased SV with time.

To our knowledge, this is the first study that describes aqueductal dynamic changes in CSF during the evolution of the disease in patients with suspected iNPH. The presenting symptoms of iNPH and a low SV are not necessarily because of atrophic changes and can show an increase in the SV with the progression of symptoms in the following months, which qualifies them as candidates for VPS. On the other hand, the progressive decrease of SV in worsening patients may be a sign of vascular injury, which may lead to the development of neurodegenerative disorders that make iNPH irreversible.

\section{Conclusion}

In patients presenting with the first signs of $\mathrm{iNPH}$, the aqueductal SV increases with the progression of the disease and then decreases again within a few months' time. This study demonstrates that patients with a low SV have not necessarily had cerebral atrophy and can show, in the following months, a progressive increase of SV that qualifies them as good candidates for VPS. The progressive reduction of the SV in untreated patients with worsening clinical symptoms may be a sign of a progressive cerebral ischemic injury, which renders NPH irreversible.

\section{References}

1. Adams RD, Fischer CM, Hakim S, et al. Symptomatic occult hydrocephalus with "normal" cerebrospinal-fluid pressure. A treatable syndrome. $N$ Engl J Med 1965;273:117-26

2. Black PM. Idiopathic normal-pressure hydrocephalus. Results of shunting in 62 patients. J Neurosurg 1980;52:371-77

3. Bergsneider M, Black PM, Klinge P, et al. Surgical management of idiopathic normal-pressure hydrocephalus. Neurosurgery 2005;57 (3 Suppl):S29-39; discussion ii-v.

4. Savolainen S, Paljärvi L, Vapalahti M. Prevalence of Alzheimer's disease in patients investigated for presumed normal pressure hydrocephalus: a clinical and neuropathological study. Acta Neurochir (Wien) 1999;141:849-53

5. Thomsen C, Ståhlberg F, Stabgaard M, et al. Fourier analysis of cerebrospinal 
fluid flow velocities: MRI Imaging study. The Scandinavian Flow Group. $R a$ diology 1990;177:659-65

6. Lee JH, Lee HK, Kim JK, et al. CSF flow quantification of the cerebral aqueduct in normal volunteers using phase contrast cine MR imaging. Korean J Radiol 2004;5:81-86

7. Bradley WG Jr, Scalzo D, Queralt J, et al. Normal-pressure hydrocephalus: evaluation with cerebrospinal fluid flow measurements at MR imaging. Radiology 1996;198:523-29

8. Folstein MF, Folstein SE, McHugh PR. "Mini-mental state". A practical method for grading the cognitive state of patients for the clinician. JPsychiatr Res 1975;12:189-98

9. Evans AJ, Iwai F, Grist TA, et al. Magnetic resonance imaging of blood flow with a phase subtraction technique. In vitro and in vivo validation. Invest Radiol 1993;28:109-15

10. Pelc LR, Pelc NJ, Rayhill SC, et al. Arterial and venous blood flow: noninvasive quantitation with MR imaging. Radiology 1992;185:809-12

11. Vanneste J, Augustijn P, Dirven C, et al. Shunting normal-pressure hydrocephalus: do the benefits outweigh the risks? A multicenter study and literature review. Neurology 1992;42:54-59

12. Vanneste J, Augustijn P, Davies GA, et al. Normal-pressure hydrocephalus. Is cisternography still useful in selecting patients for a shunt? Arch Neurol 1992;49:366-70

13. Walchenbach R, Geiger E, Thomeer RT, et al. The value of temporary external lumbar CSF drainage in predicting the outcome of shunting on normal pressure hydrocephalus. J Neurol Neurosurg Psychiatry 2002;72:503-06

14. Malm J, Kristensen B, Karlsson T, et al. The predictive value of cerebrospinal fluid dynamic tests in patients with the idiopathic adult hydrocephalus syndrome. Arch Neurol 1995;52:783-89

15. Boon AJ, Tans JT, Delwel EJ, et al. Dutch normal-pressure hydrocephalus study: prediction of outcome after shunting by resistance to outflow of cerebrospinal fluid. J Neurosurg 1997;87:687-93
16. Williams MA, Razumovsky AY, Hanley DF. Comparison of PCSF monitoring and controlled CSF drainage diagnose normal pressure hydrocephalus. Acto Neurochir Suppl 1998;71:328-30

17. Raftopoulos C, Chaskis C, Delecluse F, et al. Morphological quantitative analysis of intracranial pressure waves in normal pressure hydrocephalus. Neurol Res 1992;14:389-96

18. Bradley WG Jr, Kortman KE, Burgoyne B. Flowing cerebrospinal fluid in normal pressure hydrocephalic states: appearance on MR images. Radiology 1986;159:611-16

19. Jack CR Jr, Mokri B, Laws ER Jr, et al. MR findings in normal-pressure hydrocephalus: significance and comparison with other forms of dementia. J Comput Assist Tomogr 1987;11:923-31

20. Bradley WG Jr, Whittemore AR, Kortman KE, et al. Marked cerebrospinal fluid void: indicator of successful shunt in patients with suspected normal-pressure hydrocephalus. Radiology 1991;178:459-66

21. Mascalchi M, Arnetoli G, Inzitari D, et al. Cine-MR imaging of aqueductal CSF flow in normal pressure hydrocephalus syndrome before and after CSF shunt. Acta Radiol 1993;34:586-92

22. Bradley WG Jr. MR prediction of shunt response in NPH: CSF morphology versus physiology. AJNR Am J Neuroradiol 1998;19:1285-86

23. Nitz WR, Bradley WG Jr, Watanabe AS, et al. Flow dynamics in cerebrospinal fluid: assessment with phase-contrast velocity MR imaging performed with a retrospective cardiac gating. Radiology 1992;183:395-405

24. Fisher CM. Hydrocephalus as a cause of disturbances of gait in the elderly. Neurology 1982;32:1358-63

25. Kimura M, Tanaka A, Yoshinaga S. Significance of periventricular hemodynamics in normal pressure hydrocephalus. Neurosurgery 1992;30:701-04; discussion 704-05

26. Miyake H, Ohta T, Kajimoto Y, et al. Diamox challenge test to decide indications for cerebrospinal fluid shunting in normal pressure hydrocephalus. Acta Neurochir (Wien) 1999;141:1187-93 